\title{
Decompression sickness and echocardiography: a case of echo bubble contrast accumulation in the right ventricule \atrium.
}

\author{
Allam Harfoush ${ }^{1}$ and Mohammad Ramadan ${ }^{1}$ \\ ${ }^{1}$ Tishreen University Hospital
}

October 26, 2020

\begin{abstract}
Decompression sickness (DCS) refers to a disorder that is caused by rapid decrease of environmental pressure. Clinical manifestations are due to formation of free bubble gas as consequences of suddenly reduced ambient pressure in the various body tissues
\end{abstract}

Decompression sickness and echocardiography: a case of echo bubble contrast accumulation in the right ventricule $\backslash$ atrium.

(1)Allam Harfoush (2)Mohammad Ramadan

(1) Resident doctor at the department of cardiovascular diseases, Tishreen University Hospital (Latakia,Syria)

Contact info: +963934146934 allamharf@gmail.com

(2) Resident doctor at the department of cardiovascular diseases, Tishreen University Hospital (Latakia,Syria)

Contact info: +963992546335 dr.m93.ramadan@gmail.com

\begin{abstract}
:
Decompression sickness (DCS) refers to a disorder that is caused by rapid decrease of environmental pressure. Clinical manifestations are due to formation of free bubble gas as consequences of suddenly reduced ambient pressure in the various body tissues(1).

We describe a case of an adult scuba diver who was admitted to Tishreen University hospital with the diagnosis of Decompression sickness (DCS), the trans thorathic echo study showed spontaneous echo contrast inside of the right ventricle and the right atrium similar to agitated saline echo testing (Bubble test). However, full recovery for the previous findings was noticed within 1 hour of the hyperbaric oxygen therapy (HBOT).
\end{abstract}

\section{Key words:}

Decompression sickness, Echocardiography, Spontaneous echo contrast.

\section{Key clinical message:}

This case highlights the possible applicability of trans thorathic echocardiography in diagnosing and monitoring decompression sickness. 


\section{Introduction:}

Multiple classifications have been proposed for DCS. One approach is to divide cases as Type I or Type II. Type 1 DCS is usually characterized by musculoskeletal pain and mild cutaneous, or skin, symptoms. Type 2 symptoms are considered more consequential and typically fall into three categories: neurological and cardiopulmonary $(2)$.

This case presents a scuba diver (Fisherman) who was admitted to Tishreen University Hospital and highlights the possible applicability of echocardiography in diagnosing and monitoring DCS. However, multicenter studies are needed to evaluate the potential role of echocardiography in decompression sickness.

\section{Case report:}

A 54 scuba diver presented to Tishreen University Hospital emergency department with severe generalized arthralgia, dyspnea and lethargy after saturation diving into a 50 meter sea depth and ascended rapidly.

Patient's past medical history: moderate smoker, occasional alcoholic, no history for HTN, DMII, COPD or CKD.

Initial work-up and assessment: patient was conscious, responsive GCS $=15 \backslash 15$, however, he was mildly disoriented to time and place.

His cardiopulmonary assessment: patient was dyspneic and he mentioned the presence of a movement and breathing related chest pain with no palpitation nor syncope.

Patient also complained of generalized arthralgia which was disabling, particularly in hips, knees and elbows. Pain was bilateral, related to movement with no swelling, redness nor heat.

Physical examination: vital signs: Blood pressure $=90 \backslash 60 \mathrm{mmhg}$, Pulse $=95 \backslash \mathrm{m}$, saturation $=90 \%$, Respiratory rate $=24 \backslash \mathrm{m}$, Temperature $=36.6$

Speech, facial symmetry, motor and sensory function, reflex activity and the rest of the emergency neurology assessment was within normal.

Cardiac and lungs auscultation: within normal limits.

Abdominal palpation: within normal limits.

According to the history, clinical picture and physical examination; a differential diagnosis was made:

1. Pmeumothorax

2. Decompression sickness

3. Pulmonary embolism

\section{Investigations:}

1. ECG : within normal limits. (Figure1)

2. CXR : within normal limits. (Figure2)

3. Blood tests :

- ABG: metabolic acidosis: $\mathrm{PH}=7.325, \mathrm{PCO} 2=28.6 \mathrm{mmhg}, \mathrm{HCO}=14.9 \mathrm{mmol} \backslash \mathrm{l}, \mathrm{PO} 2=63 \mathrm{mmhg}$, SPO2 $=90 \%$.

- $\mathrm{CBC}: \mathrm{WBC}=13.5, \mathrm{RBC}=5, \mathrm{HGB}=14.3, \mathrm{MCV}=82, \mathrm{PLT}=232$.

- Chemistry panel: Crea $=0.9 \mathrm{mg} \backslash \mathrm{dl}, \quad$ Urea $=25.15 \mathrm{mg} \backslash \mathrm{dl}, \quad \mathrm{CRP}=73.3 \mathrm{mg} \backslash \mathrm{l}, \quad \mathrm{ESR}=11 \mathrm{~mm} \backslash \mathrm{hr}$, $\mathrm{CK}=194.6 \mathrm{u} \backslash \mathrm{l}, \mathrm{CK}-\mathrm{MB}=26 \mathrm{u} \backslash \mathrm{l}, \mathrm{NA}=146 \mathrm{mmol} \backslash \mathrm{l}, \mathrm{K}=3,9 \mathrm{mmol} \backslash \mathrm{l}$.

- $\mathrm{D}$-dimer $=385 \mathrm{ng} \backslash \mathrm{ml}$ (Reference range 0-500 $\mathrm{ng} \backslash \mathrm{ml}$ ).

Trans-thorathic echocardiography TTE : 
Normal left ventricular function, no valve diseases, normal size right ventricle and normal function. However: there was spontaneous echo contrast inside of the right ventricle and the right atrium similar to agitated saline echo testing (Bubble test).

During the exam, patient was asked to start the valsalva maneuver to assess the presence of P.F.O (Patent Foramen Ovale), and there was no flow.

With such symptoms, examination and investigations, the diagnosis of decompression sickness type II was the most convenient.

He was admitted for follow-up with medication management plan of:

1. Nasal cannula Oxygen (3 $\mathrm{l} \backslash \mathrm{m})$.

2. Left lateral decubitis.

3. I.V saline serum $(100 \mathrm{ml} \backslash \mathrm{h})$.

4. S.C Enoxiparin (40m.g qDay).

Meanwhile, an immediate contact with the center of hyperbaric oxygen therapy (HBOT) was made, and the first session was made within $18 \mathrm{~h}$ of admission.

Post hyperbaric oxygen therapy (HBOT) session:

Clinical and hymodynamic improvement was immediately noticed with saturation $=98 \%$ on room air, blood pressure $=135 \backslash 75 \mathrm{mmhg}, \mathrm{HR}=75 \backslash \mathrm{m}, \mathrm{RR}=17 \backslash \mathrm{m}$. Together with a gradual recovery of his neurological symptoms (related to disorientation).

Transthorathic echocardiography was repeated within one hour of oxygen therapy (HBOT) session in order to monitor the findings of the first study (Spontaneous echo contrast inside of the right ventricle and the right atrium similar to agitated saline echo testing), which was fully disappeared with no additional pathological findings.

Patient was discharged within $48 \mathrm{~h}$ of admission after two sessions of hyperbaric oxygen therapy (HBOT).

Upon discharge: patient was almost symptoms free excluding mild arthralgia alleviated with paracetamol.

Patient was reevaluated 15 days post-discharge: no symptoms or complaints were mentioned.

\section{Discussion:}

Increasing depth leads nitrogen in compressed air to cross through the alveoli into the blood. Increasing amounts of nitrogen dissolve and accumulate in the tissues(3).

Ascending too quickly after diving causes the dissolved nitrogen to return to its gas form while still in the blood or tissues, causing bubbles to form(3)(4).

Decompression-induced tissue bubbles can be detected using Dual-Frequency Ultrasound (DFU), which also could be used to monitor micro-bubbles at multiple sites on the body(5).

This case present the usage of transthorathic echo as a bubbles detecting tool, together with its potential role in monitoring after hyperbaric oxygen therapy (HBOT).

\section{ACKNOWLEDGEMENTS}

None.

\section{CONFLICT OF INTEREST STATEMENT}

None. 


\section{FUNDING}

None.

\section{ETHICAL APPROVAL}

Not applicable.

\section{GUARANTOR}

I will accept full responsibility for the work.

\section{REFERENCES}

1. Severe Decompression Sickness in Divers. 1999;151(5-6):111-6.

2. The probability and severity of decompression sickness. 2017 Mar 15. doi: 10.1371/journal.pone.0172665.

3. Brubakk A, Neuman T. Bennett and Elliott's physiology and medicine of diving. 5th ed 2003, Elsevier.

4. Microbubbles are detected prior to larger bubbles following decompression. J. G. Swan, J. C. Wilbur, K. L. Moodie, S. A. Kane, D. A. Knaus, S. D. Phillips, T.L. Beach, A. M. Fellows, P. J. Magari and J. C. Buckey doi:10.1152/japplphysiol.01156.2013

5. Microbubble detection following hyperbaric chamber dives using dual-frequency ultrasound J G Swan 1, B D Bollinger, T G Donoghue, J C Wilbur, S D Phillips, D L Alvarenga, D A Knaus, P J Magari, J C Buckey DOI: 10.1152/japplphysiol.01203.2010.

Figure 1 : Patient's ECG: normal sinus rhythm, heart rate=92, no ST elevation, no conduction disturbance.

Figure 2 : Patient's CXR: normal cardiothorathic ratio, no signs of pneumothorax, atelectasis or consolidation

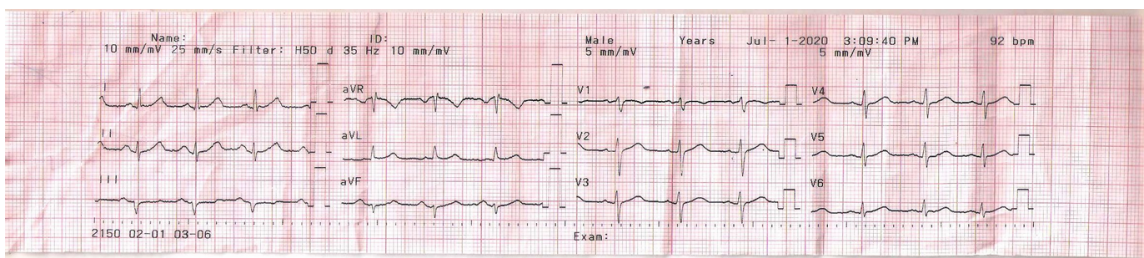




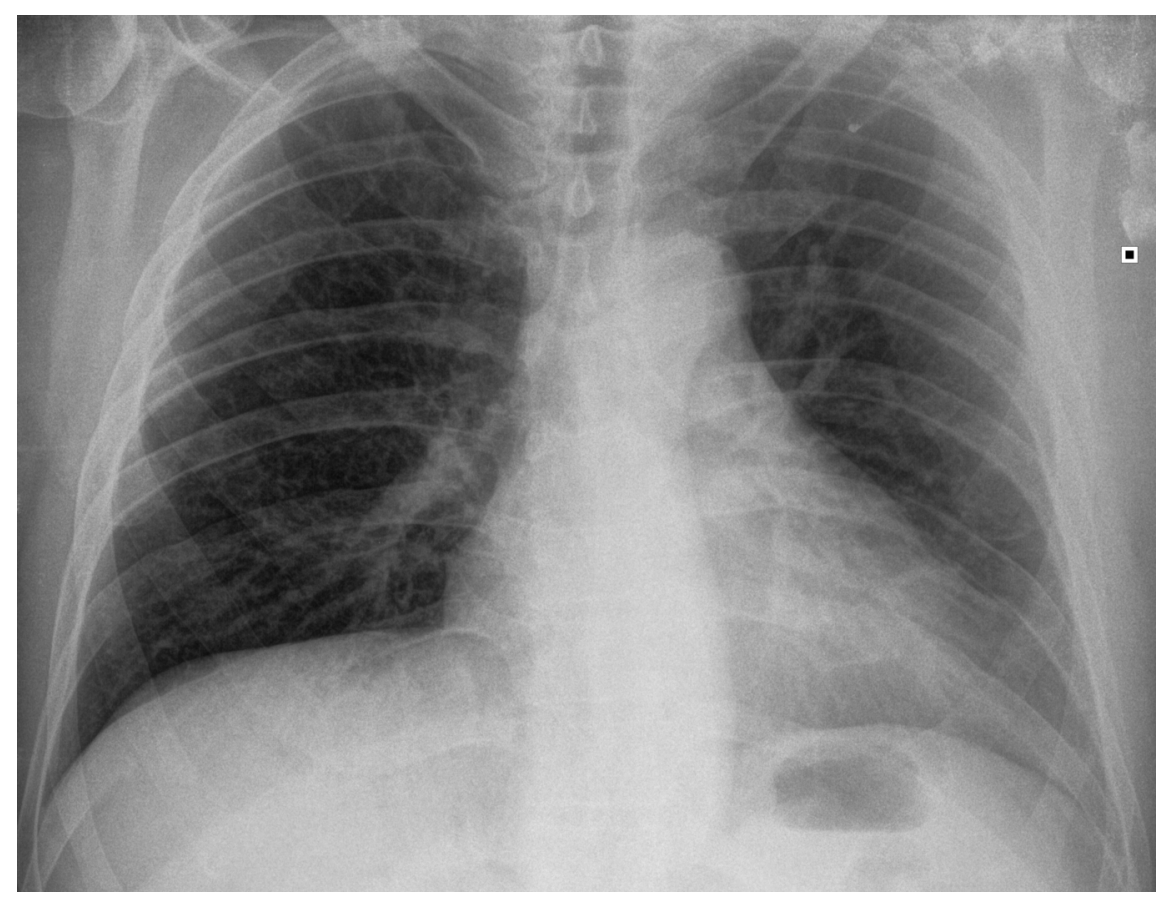

